\title{
BEAL CONJECTURE
}

\section{Slobodan Stanojevic}

12 Stayner Court, Chelsea VIC 3196

Melbourne, Victoria, Australia

Stanb9@optusnet.com.au

Keywords and phrases: Beal Conjecture, Fermat's Last Theorem, Analysis

\section{Abstract}

Beal Conjecture was formulated in 1997 and presented as a generalization

Theorem, within the field of number theory.

It states that, if:

$$
-A^{x}+B^{y}=C^{z}
$$

$-A, B, C, x, y$, and $z$ are positive integers, and $x, y$, and $z>2$

-Then $A, B$ and $C$ must have a common prime factor.

This article presents solution and the proof for the Beal Co yre.

\section{Introduction}

The aim of this paper is to provide proof of Beal Conject with th use of basic mathematics.

This article is divided into three parts:

The first part presents two theorems

Theorem 1: Any two of exponent $y$, at $\mathrm{z}$ must the same.

Theorem 2: The factors A, B aryonon prime factor.

The second part presents 1 Jutions an of Beal Conjecture.

The third part include anal, and discussion on topic of the solutions of Beal Conjecture including some exa rpies.

\section{THEOREMS}

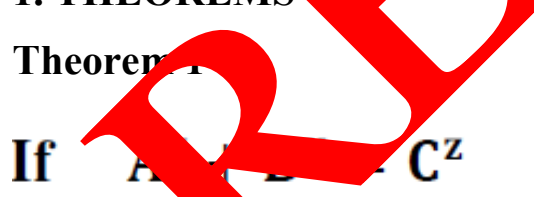

$A, B, C, x$, nd $z$ are positive integers and $x, y, z>2$

$A, B$ and $C$ in st have a common prime factor.

Then any two of exponents $x, y$, or $z$ must be the same.

\section{Proof}

The exponents $\mathrm{x}, \mathrm{y}$ and $\mathrm{z}$ we can express as: 
The exponents $\mathrm{x}, \mathrm{y}$ and $\mathrm{z}$ we can express as:

$\begin{array}{ll}\mathrm{x}=\mathrm{f}_{1}(\mathrm{n}) ; \mathrm{x}=\mathrm{n} & \mathrm{x}, \mathrm{y} \text { and } \mathrm{z}>2 \\ \mathrm{y}=\mathrm{f}_{2}(\mathrm{n}) ; \mathrm{y}=\mathrm{n}+\mathrm{u} & \mathrm{n}>2 \text { or } \mathrm{n} \geq 3 \\ \mathrm{z}=\mathrm{f}_{3}(\mathrm{n}) \quad ; \mathrm{z}=\mathrm{n}+\mathrm{v} & 0 \leq \mathrm{u}, \mathrm{v}<\mathrm{n}\end{array}$

Then:

$\mathrm{A}^{\mathrm{x}}+\mathrm{B}^{\mathrm{y}}=\mathrm{C}^{\mathrm{z}}$

$A^{n}+B^{n+u}=C^{n+v}$

Let as consider following cases:
1. $\mathrm{u}=\mathrm{v}=0$
2. $\mathrm{u} \neq \mathrm{v}$
$\mathrm{x}=\mathrm{y}=\mathrm{z}=\mathrm{n}$
$\mathrm{x} \neq \mathrm{y} \neq \mathrm{z}$
3. $\mathrm{u} \neq \mathrm{v}$
$0 \leq \mathrm{u}, \mathrm{v}<\mathrm{n}$,
$0 \leq \mathrm{u}, \mathrm{v}<2$
( $\mathrm{u}$ or $\mathrm{v}$ or both $\geq 2$ )

Case 1. For $u=v=0 ; x=y=z=n$

We get the Fermat's Last Theorem

$\mathrm{A}^{\mathrm{n}}+\mathrm{B}^{\mathrm{n}}=\mathrm{C}^{\mathrm{n}}$

It has no integer solutions for $\mathrm{n}>2$.

Case 2. For $\mathrm{u} \neq \mathrm{v} ; \mathrm{x} \neq \mathrm{y} \neq \mathrm{z} ; 0 \leq \mathrm{u}, \mathrm{v}<\mathrm{n}$, in case ( $\mathrm{u}$ or or both $\geq 2$ ) we get:

$\mathbf{A}^{\mathbf{n}}+\mathbf{B}^{\mathbf{n}+\mathbf{u}}=\mathbf{C}^{\mathbf{n + v}}$

If $\mathrm{A}, \mathrm{B}$ and $\mathrm{C}$ have a common prime factor $\mathrm{N}$, th them express them as:

$\mathrm{A}=\mathrm{KN}$

$\mathrm{B}=\mathrm{PN}$

$\mathrm{C}=\mathrm{SN}$

$\mathrm{K}, \mathrm{P}$, and $\mathrm{S} \geq 1$ and positive intes

$\mathrm{N}$ Common prime factor or $\mathrm{C}$. hon stor, trun we can write:

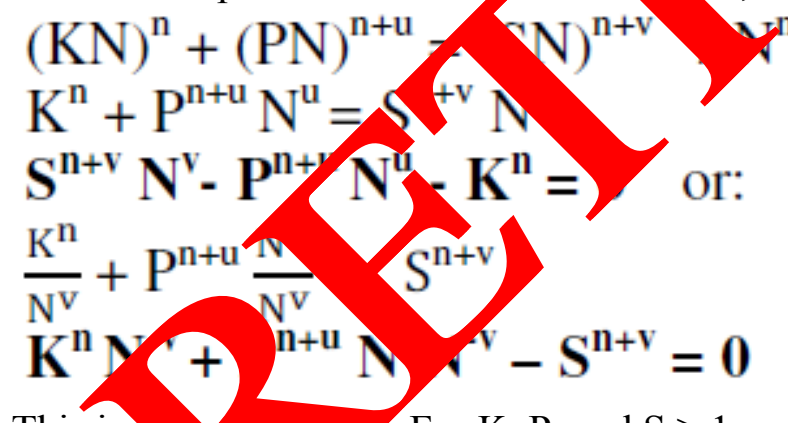

This is $\mathrm{C}$ For $\mathrm{K}, \mathrm{P}$, and $\mathrm{S} \geq 1$ and $\mathrm{n} \geq 3$,

than in cas $<\mathrm{u}, \mathrm{v}<\mathrm{n},(\mathrm{u}$ or $\mathrm{v}$ or both $\geq 2 ; \mathrm{u} \neq \mathrm{v}$ ) it is not possible that common prime factor $\mathrm{N}$ and $\mathrm{A}, \mathrm{B}$, and are all integers simultaneously, except for the particular case where: 


$$
\begin{aligned}
& \frac{\mathrm{V}=2}{\mathrm{~K}=2} ; \underline{\mathrm{u}=0} ; \mathrm{P}=1 ; \mathrm{n}=3 \\
& \\
& \mathrm{~A}^{\mathrm{x}}+\mathrm{B}^{\mathrm{y}}=\mathrm{C}^{\mathrm{z}} \\
& (\mathbf{K N})^{\mathrm{n}}+(\mathbf{P N})^{\mathrm{n}+\mathrm{u}}=(\mathbf{S N})^{\mathrm{n}+\mathrm{v}} \\
& \mathrm{K}^{\mathrm{n}} \mathrm{N}^{\mathrm{n}}+\mathrm{N}^{\mathrm{n}}=\mathrm{N}^{\mathrm{n}+\mathrm{v}} \\
& \mathrm{K}^{3} \mathrm{~N}^{3}+\mathrm{N}^{3}=\mathrm{N}^{3+2} \quad /: \mathrm{N}^{3} \\
& \mathrm{~K}^{3}+1=\mathrm{N}^{2} \\
& 2^{3}+1=\mathrm{N}^{2} \\
& \mathrm{~N}^{2}=9 ; \\
& \mathrm{N}=3-\mathrm{Common}^{3} \text { prime factor } \\
& \mathrm{K}^{3} \mathrm{~N}^{3}+\mathrm{N}^{3}=\mathrm{N}^{3+2} \\
& 2^{3} 3^{3}+3^{3}=3^{3+2} \\
& 6^{3}+3^{3}=3^{5}
\end{aligned}
$$

Case 3: $\mathrm{u} \neq \mathrm{v} ; 0 \leq \mathrm{u}, \mathrm{v}<2$

Then: $\mathrm{u}, \mathrm{v}=\{0,1\}$

a. $\mathrm{u}=0 ; \mathrm{x}=\mathrm{y}=\mathrm{n}$

$\mathrm{v}=1 ; \mathrm{z}=\mathrm{n}+1$

b. $\mathrm{u}=1 ; \mathrm{x}=\mathrm{z}=\mathrm{n}$

$\mathrm{v}=0 ; \mathrm{y}=\mathrm{n}+1$

If we substitute in equation:

$$
\begin{aligned}
& A^{x}+B^{y}=C^{z} \\
& A^{n}+B^{n+u}=C^{n+v}
\end{aligned}
$$

We then have:

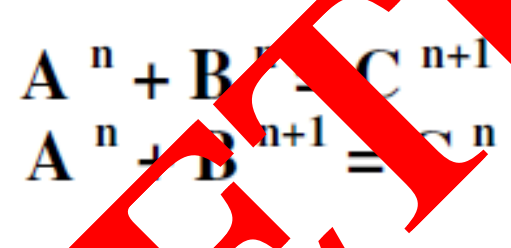

Based on the equ in (a), ) and Beal Conjecture:

If $A^{x}-B^{y}=z^{z}$

$A, B, x, y, d z$ are ositive integers and $x, y$, and $z>2$.

$A, B$ an a common prime factor,

Then $x, y$, d $z$ can never be all equal or different simultaneously. Any two of $x, y$, and $z$ must be the s.

Theorem 2

$$
\text { If } A^{x}+B^{y}=C^{z}
$$

$A, B, C, x, y$ and $z$ are positive integers and $x, y, z>2$.

Then A, B and C must have a common prime factor "Ni" or common factor $\mathbf{N}$.

\section{Proof}

If we start from the opposite assumption for Beal Conjecture $\mathrm{A}^{\mathrm{x}}+\mathrm{B}^{\mathrm{y}}=\mathrm{C}^{\mathrm{z}}$ that: 
$\mathrm{A}, \mathrm{B}$ and $\mathrm{C}$ have no common prime factor.

1. $\mathrm{A}, \mathrm{B}$ and $\mathrm{C}$ are all different prime factors $\mathrm{A} \neq \mathrm{B} \neq \mathrm{C}$. E.g. 2, 3, 5, 7, $11 \ldots$

2. Or different common factors with no common prime factors.

E.g. $\mathrm{A}=2 \times 11, \mathrm{~B}=3 \times 7$, and $\mathrm{C}=5 \times 13 \times 17$.

Then following Theorem 1

(a) $\mathrm{A}^{\mathrm{n}}+\mathrm{B}^{\mathrm{n}}=\mathrm{C}^{\mathrm{n}+1}$

(b) $\mathrm{A}^{\mathrm{n}}+\mathrm{B}^{\mathrm{n}+1}=\mathrm{C}^{\mathrm{n}}$

We have:

(a) $\mathrm{A}^{\mathrm{n}}+\mathrm{B}^{\mathrm{n}}=\mathrm{C}^{\mathrm{n}} \mathrm{C} \quad /: \mathrm{C}^{\mathrm{n}}$

(b) $\mathrm{A}^{\mathrm{n}}+\mathrm{B}^{\mathrm{n}} \mathrm{B}=\mathrm{C}^{\mathrm{n}} /: \mathrm{B}^{\mathrm{n}}$

(a) $\frac{\mathrm{A}^{\mathrm{n}}}{\mathrm{C}^{\mathrm{n}}}+\frac{\mathrm{B}^{\mathrm{n}}}{\mathrm{C}^{\mathrm{n}}}=\mathrm{C}$

(b) $\frac{\mathrm{C}^{\mathrm{n}}}{\mathrm{B}^{\mathrm{n}}}-\frac{\mathrm{A}^{\mathrm{n}}}{\mathrm{B}^{\mathrm{n}}}=\mathrm{B}$

1. If $\mathrm{A}, \mathrm{B}$ and $\mathrm{C}$ are all different prime factors $\mathrm{A} \neq \mathrm{B} \neq$ potient $\mathrm{f}$ two distinct prime numbers is always irrational or rational (but not a positi integer), factor is divisible only by 1 and themselves, then any one factor of A, B or C irrational or rational but can't be positive integer.

2. If any two of A, B, and C are co orime each o $y$ and positive integers, then third one can't be

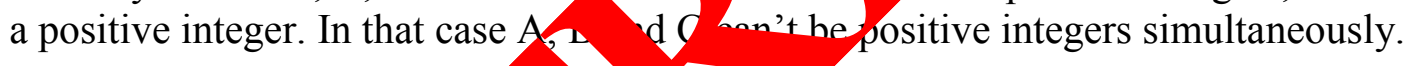

3. From equation (a), C

$\frac{\mathrm{A}^{\mathrm{n}}}{\mathrm{C}^{\mathrm{n}}}$ and $\frac{\mathrm{B}^{\mathrm{n}}}{\mathrm{C}^{\mathrm{n}}}$ will be ositive integers

$\mathrm{A}$ and $\mathrm{B}$ is divic le by rellows:

$\mathrm{A}=\mathrm{KC} ; \mathbf{B}=\mathbf{P C}$

Where $\mathrm{K}$ are $\mathrm{P}$ ive iteger $\geq 1$;

$\left.\frac{\mathrm{A}}{\mathrm{C}}=\frac{\mathrm{K} C}{\mathrm{C}}=\mathrm{K}\right)$ or

$\frac{\mathrm{B}}{\mathrm{C}}=\frac{\mathrm{PC}}{\mathrm{C}}=$

If two of $A, B$ and $C$ are positive integers and have common prime factor, then third one must have common prime factor too.

Then equation (a) $A^{n}+B^{n}=C^{n} C$ for: $A=K C$ and $B=P C$ becomes:

(a) $(\mathrm{KC})^{\mathrm{n}}+(\mathbf{P C})^{\mathrm{n}}=\mathrm{C}^{\mathrm{n}+1}$

Where $\mathbf{C}=\mathbf{N}$ is a common factor

$\mathrm{K}$ and $\mathrm{P}$ are positive integers $\geq 1$ 
We can show the same for equation $(\mathbf{b}) \mathrm{A}^{\mathrm{n}}+\mathrm{B}^{\mathrm{n}} \mathrm{B}=\mathrm{C}^{\mathrm{n}}$ for $\mathrm{A}=\mathrm{KB}$ and $\mathrm{C}=\mathrm{SB}$

(b) $(\mathrm{KB})^{\mathrm{n}}+\mathbf{B}^{\mathrm{n}+1}=(\mathrm{SB})^{\mathrm{n}}$

Where $\mathbf{B}=\mathbf{N}$ is common factor

$\mathrm{K}$ and $\mathrm{S}$ are positive integers $\geq 1$

Then equation (a) and (b) becomes:

(1) $(\mathrm{KN}) \mathrm{n}+(\mathrm{PN}) \mathrm{n}=\mathrm{N} \mathbf{n}+1$

(2) $(\mathrm{KN}) \mathrm{n}+\mathrm{N} \mathbf{n}+\mathbf{1}=(\mathrm{SN}) \mathrm{n}$

From the above we can conclude that any one of $A, B$ and $C$ must be the commonfacto elf.

Based on the equations (1) and (2) and Beal Conjecture if: $A^{x}+B^{y}=C^{z}$

$A, B, C, x, y$ and $z$ are positive integers, and $x, y, z>2$ we have pros that flo fo s of $A, B$ and $\mathrm{C}$ must have a common prime factor $\mathrm{Ni}$ or common factor $\mathrm{N}$ en value $\geq 3$, and $\mathrm{K}$, $P, S$ positive integers $\geq 1$

\section{PROOF THE BEAL CONJECTURE}

Based on the theorems 1 and 2 we have:

From equation (1):

$$
\begin{aligned}
& \mathbf{K}^{\mathrm{n}} \mathbf{N}^{\mathrm{n}}+\mathbf{P}^{\mathrm{n}} \mathbf{N}^{\mathrm{n}}=\mathbf{N}^{\mathrm{n}+1} \\
& \mathrm{~N}^{\mathrm{n}}\left(\mathrm{K}^{\mathrm{n}}+\mathrm{P}^{\mathrm{n}}\right)=\mathrm{N}^{\mathrm{n}} \mathrm{N}
\end{aligned}
$$

And from equation ( 2):

$$
\begin{aligned}
& \mathbf{K}^{\mathrm{n}} \mathbf{N}^{\mathrm{n}}+\mathbf{N}^{\mathrm{n}+1} \\
& \mathrm{~N}^{\mathrm{n}}\left(\mathrm{K}^{\mathrm{n}}+\mathrm{N}\right.
\end{aligned}
$$$$
\mathbf{N}=\mathbf{S}^{\mathrm{n}}
$$

These are solut ins an proof of 2 ne Beal Conjecture for any value of:

$\mathrm{K}, \mathrm{P}, \mathrm{S} \geqslant \mathrm{L}, \mathrm{K}, \mathrm{N}=\{1,2,3,4 \ldots\}$

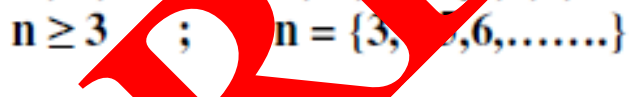

1- Substil 7 in equation (1), (1.1), (2), (2.1) any value of K,P,S $\geq 1$ and $n \geq 3$ we obtain infinite number of so ons of the Beal Conjecture.

$$
\begin{array}{ll}
\text { E.g. } 1 & \mathrm{n}=4 ; \mathrm{K}=3 ; \mathrm{P}=2 \\
\mathrm{~N}=\mathrm{K}^{\mathbf{n}}+\mathbf{P}^{\mathbf{n}}=3^{4}+2^{4}=97 \\
\mathrm{~K}^{\mathrm{n}} \mathbf{N}^{\mathrm{n}}+\mathbf{P}^{\mathrm{n}} \mathbf{N}^{\mathbf{n}}=\mathbf{N}^{\mathbf{n}+1} \\
3^{4} 97^{4}+2^{4} 97^{4}=97^{5} \\
291^{4}+194^{4}=\mathbf{9 7}
\end{array}
$$

$$
\begin{aligned}
& \text { E.g. } 2 \mathrm{n}=3 ; \mathrm{S}=5 ; \mathrm{K}=3 \\
& \mathbf{N}=\mathbf{S}^{\mathbf{n}}-\mathbf{K}^{\mathbf{n}}=5^{3}-3^{3}=98 \\
& \mathrm{~K}^{\mathrm{n}} \mathrm{N}^{\mathrm{n}}+\mathrm{N}^{\mathrm{n}+1}=\mathrm{S}^{\mathrm{n}} \mathrm{N}^{\mathrm{n}} \\
& 3^{3} 98^{3}+98^{4}=5^{3} 98^{3} \\
& 294^{3}+98^{4}=490^{3}
\end{aligned}
$$


2- Also if we multiple with common factor $\mathrm{N}^{\mathrm{nt}}$ equations (1) and (2), $\mathrm{t}=\{1,2,3, .$.$\} , or multiple or$ divide with common prime factor $\mathrm{Ni}^{\mathrm{n}}$, where

$\mathrm{N}=\mathrm{N}_{1} \mathrm{x} \mathrm{N}_{2} \mathrm{x} \ldots$, and $\mathrm{N}_{1}=\mathrm{N}_{2}=\ldots$

E.g $\mathrm{N}=8=2 \times 2 \times 2=\mathrm{N}_{1} \mathrm{~N}_{2} \mathrm{~N}_{3} ; \mathrm{N}_{1}=\mathrm{N}_{2}=\mathrm{N}_{3}$, or

$\mathrm{N}=18=3 \times 3 \times 2=\mathrm{N}_{1} \mathrm{~N}_{2} \mathrm{~N}_{3}$

We get a different form of the same equations.

E.g $\mathrm{K}=2 \quad \mathrm{P}=1 \quad \mathrm{n}=3$

$$
\begin{aligned}
& \mathbf{K}^{\mathbf{n}} \mathbf{N}^{\mathbf{n}}+\mathbf{P}^{\mathbf{n}} \mathbf{N}^{\mathbf{n}}=\mathbf{N}^{\mathbf{n}+1} \\
& \mathbf{N}=\mathbf{K}^{\mathbf{n}}+\mathbf{P}^{\mathbf{n}} \\
& \mathrm{N}=2^{3}+1^{3} \\
& \mathrm{~N}=9 \\
& 2^{3} 9^{3}+9^{3}=9^{4}
\end{aligned}
$$

E.g $1 \quad 2^{3} 9^{3}+9^{3}=9^{4} \quad / 9^{3}$

$2^{3} 9^{6}+9^{6}=9^{7}$

$$
162^{3}+81^{3}=9^{7}
$$

E.g $22^{3} 9^{3}+9^{3}=9^{4}$ or

$18^{3}+9^{3}=9^{4} / 3^{3}$

$2^{3} 3^{3} 3^{3}+3^{3} 3^{3}=3^{4} 3^{4} / 3^{3}$

$54^{3}+27^{3}=3^{11}$

E.g $3 \quad 18^{3}+9^{3}=9^{4} /: 3^{3}$

$2^{3} 3^{3} 3^{3}+3^{3} 3^{3}=3^{4} 3^{4} \quad 1: 3^{3}$

$6^{3}+3^{3}=3^{5}$

3- If we substitute the value

E.g: $3^{9}=27^{3}$ or $3^{6}=9^{3}$ or $2^{8}=4^{4}$ or $2^{9}=$

Then we get a different $f$,rm the same, 1 uations.

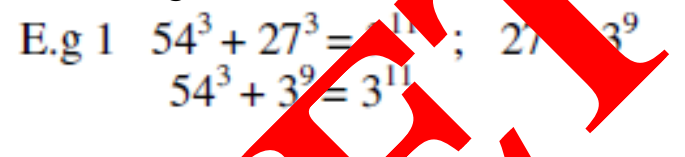

E.g $218^{3}+9^{3} ; 9^{3}=3^{6}$

18. $3^{6}=$

$18^{3}-3^{6}=3^{8} ; 9^{4}=3^{8}$

E.g $3 \quad 2^{8}=2^{9} \quad ; \quad 2^{8}=4^{4}$

$4^{4}+2^{8}=8^{3} \quad ; \quad 2^{9}=8^{3}$

$4^{4}+4^{4}=8^{3}$

1. Following above and equations (1), (1.1), (2), and (2.1), for $K, P$, and $S \geq 1$ and $n \geq 3$, we obtain infinite number of solutions of the Beal Conjecture.

2. If we multiple equations (1) and (2), with common factor $\mathrm{N}^{\text {tn }}$ or multiply or divide with common prime factor $\mathrm{Ni}^{\mathrm{tn}}$, as shown, $\mathrm{t}=\{1,2,3, \ldots\}$, we get a different form of the same equations.

3. If we substitute the value of any factor of $\mathrm{A}, \mathrm{B}$ or $\mathrm{C}$ in different form, as shown, $\mathrm{Eg} .\left(2^{9}=\right.$ $8^{3}$ ); we get a different form of the same equations. This is a solution and proof of Beal Conjecture. 


\section{ANALYSIS AND DISCUSSION}

\section{A. ANALYSIS WHEN “N” IS COMON FACTOR N = N1 x N2 x...}

If $\mathrm{Ax}+\mathrm{By}=\mathrm{Cz}$

Where $\mathrm{A}, \mathrm{B}$ and $\mathrm{C}$ are positive integers and have a common factor $\mathrm{N}$, we can write:

$\mathrm{A}=\mathrm{KN} \mathrm{N}=\mathrm{N} 1 \times \mathrm{N} 2 \times \ldots$

$\mathrm{B}=\mathrm{PN} \mathrm{Ni}=\mathrm{N} 1$ or $\mathrm{N} 2$ or....

$\mathrm{C}=\mathrm{SN}$

Where Ni is:

1. Common prime factor such as:

$\mathrm{Ni}=\{2,3,5,7,11,13,17,19, \ldots\}$

Where $\mathrm{N}$ is:

2. Common factor which contains common prime factor such as:

$\mathrm{N}=65=5 \times 13=\mathrm{N} 1 \times \mathrm{N} 2$ or,

$\mathrm{N}=175=5 \times 5 \times 7=\mathrm{N} 1 \mathrm{~N} 2 \mathrm{~N} 3$

3. $\mathrm{K}, \mathrm{P}, \mathrm{S}$ positive integers $\geq 1$

$\mathrm{K}, \mathrm{P}, \mathrm{S}=\{1,2,3,4, \ldots \ldots\}$

Then we can write:

$(\mathrm{KN}) \mathrm{X}+(\mathrm{PN}) \mathrm{Y}=(\mathrm{SN}) \mathrm{Z}$

Where $\mathrm{N}=\mathrm{N}_{1} \mathrm{~N}_{2}$ is common factor and $\mathrm{N}_{1}$ and $\mathrm{N}_{2}$ are common prime factors We then have:

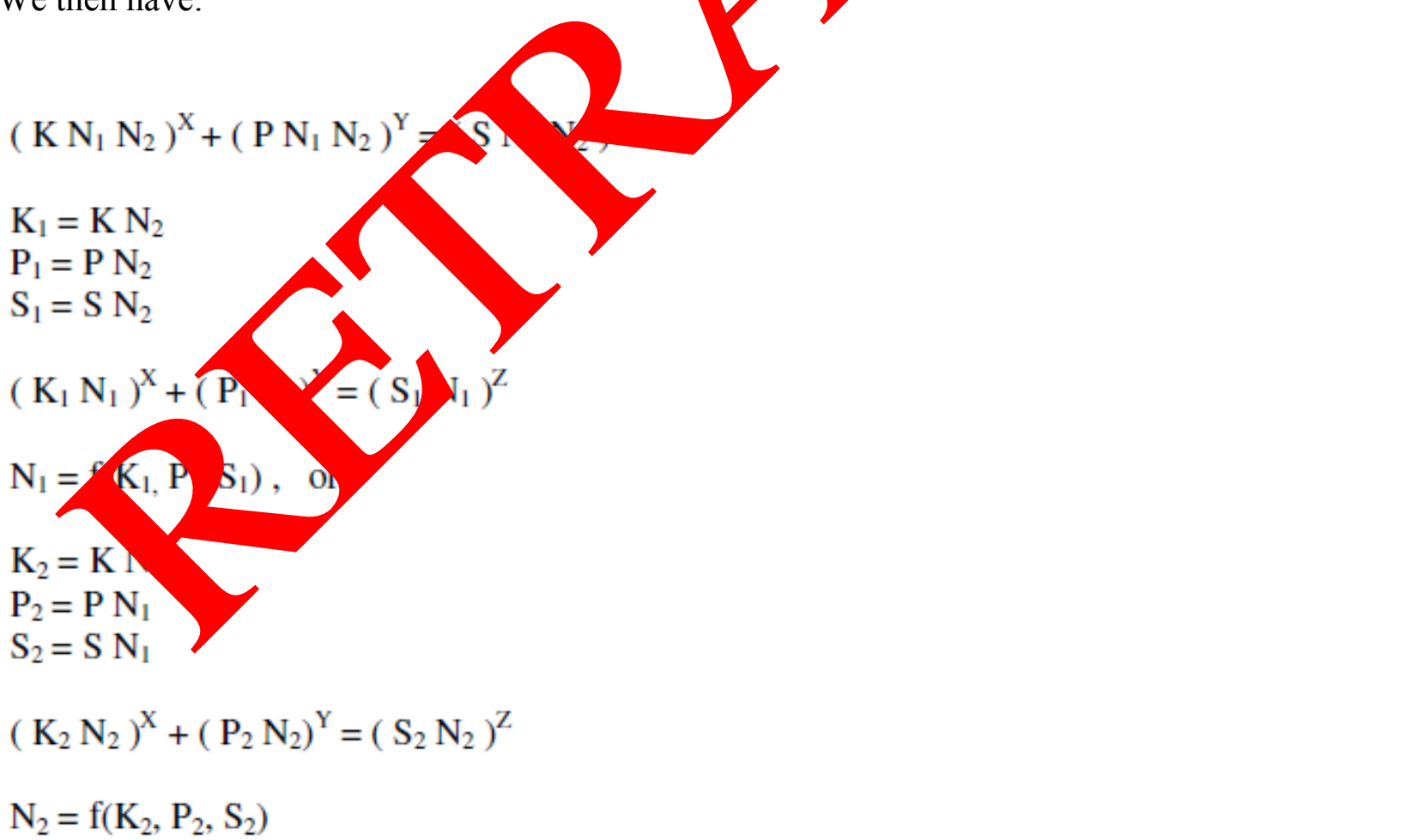

If the exponents $\mathrm{x}, \mathrm{y}$ and $\mathrm{z}$ are positive integers, all greater than 2 , we can express them as: 


$$
\begin{array}{ll}
\mathrm{x}=\mathrm{f}_{1}(\mathrm{n}), & \mathrm{y}=\mathrm{f}_{2}(\mathrm{n}) \text { and } \mathrm{z}=\mathrm{f}_{3}(\mathrm{n}) \\
\mathrm{x}=\mathrm{n} & \mathrm{x}, \mathrm{y} \text { and } \mathrm{z}>2 \\
\mathrm{y}=\mathrm{n}+\mathrm{u} & \mathrm{n} \geq 3 \text { or } \mathrm{n}>2 \\
\mathrm{z}=\mathrm{n}+\mathrm{v} & 0 \leq \mathrm{u}, \mathrm{v}<\mathrm{n}
\end{array}
$$

If we substitute the expression for $\mathrm{A}, \mathrm{B}, \mathrm{C}, \mathrm{x}, \mathrm{y}$ and $\mathrm{z}$ in Beal conjecture we obtain the following form of the Beal conjecture.

$$
(\mathrm{KiNi})^{\mathrm{n}}+(\mathrm{PiNi})^{\mathrm{n}+\mathrm{u}}=(\mathrm{SiNi})^{\mathrm{n}+\mathrm{v}}
$$

From this equation we can calculate a common prime factor $\mathrm{Ni}$ for every value of the in ated conditions.

$\mathrm{n} \geq 3$

$\mathrm{Ki}, \mathrm{Pi}, \mathrm{Si} \geq 1$ and

$\mathrm{u}, \mathrm{v}=\{0,1\} \quad ; \mathrm{u} \neq \mathrm{v}$

For: $\quad \mathrm{u} \neq \mathrm{v}$

$$
\mathrm{u}, \mathrm{v}=\{0,1\}
$$

a) $\mathrm{u}=0 \quad ; \quad \mathrm{x}=\mathrm{y}=\mathrm{n}$

$\mathrm{v}=1 \quad ; \quad \mathrm{z}=\mathrm{n}+1$

We get:

(3)

$$
(\mathrm{KiNi})^{\mathrm{n}}+(\mathrm{PiNi})^{\mathrm{n}}=(\mathrm{SiNi})
$$

$$
\mathrm{Ni}=\frac{\mathrm{Ki}^{\mathrm{n}}+\mathrm{Pi}^{\mathrm{n}}}{\mathrm{Si}^{\mathrm{n}+1}}
$$

b) $\mathrm{u}=1$

$$
\mathrm{v}=0
$$

We get:

(4)

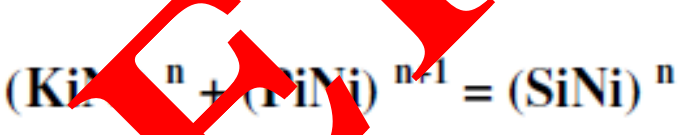

$$
\mathrm{i}=\frac{\mathrm{Si}}{\mathrm{Di}^{1+1}} \quad \mathrm{Si}>\mathrm{Ki}
$$

E.g $\mathrm{n}=3 ; \quad / 3 ; \mathrm{P}=1$

$\mathbf{N}=\mathbf{K}^{\mathbf{n}}+\mathbf{P}^{\mathbf{n}}=3^{3}+1^{3}=28$

$\mathrm{K}^{\mathrm{n}} \mathrm{N}^{\mathrm{n}}+\mathrm{P}^{\mathrm{n}} \mathrm{N}^{\mathrm{n}}=\mathrm{N}^{\mathrm{n}+1}$

$3^{3} 28^{3}+28^{3}=28^{4}$

$84^{3}+28^{3}=28^{4}$

$\mathrm{N}=28$ is common factor

$\mathrm{N}=2 \times 2 \times 7=\mathrm{N}_{1} \times \mathrm{N}_{2} \times \mathrm{N}_{3}$

$\mathrm{N}_{1}=2, \mathrm{~N}_{2}=2, \mathrm{~N}_{3}=7$ is common prime factor. And we get the same expression if we write: 


$$
\begin{aligned}
& 3^{3} \mathrm{~N}_{1}{ }^{3} \mathrm{~N}_{2}{ }^{3} \mathrm{~N}_{3}{ }^{3}+\mathrm{N}_{1}{ }^{3} \mathrm{~N}_{2}{ }^{3} \mathrm{~N}_{3}{ }^{3}=\mathrm{N}_{1}{ }^{4} \mathrm{~N}_{2}{ }^{4} \mathrm{~N}_{3}{ }^{4} \\
& 3^{3} 2^{3} 2^{3} 7^{3}+2^{3} 2^{3} 7^{3}=2^{4} 2^{4} 7^{4} \\
& \mathrm{~N}_{1}=2 ; \mathrm{N}_{2}=2 ; \mathrm{N}_{3}=7 \\
& \mathrm{~K}_{1}=3 \mathrm{~N}_{1} \mathrm{~N}_{2}=3 \times 2 \times 2=12 \\
& \mathrm{P}_{1}=\mathrm{N}_{1} \mathrm{~N}_{2}=2 \times 2=4 \\
& \mathrm{~S}_{1}=\mathrm{N}_{1} \mathrm{~N}_{2}=2 \times 2=4
\end{aligned}
$$

$$
(\mathrm{KiNi})^{\mathrm{n}}+(\mathrm{PiNi})^{\mathrm{n}}=(\mathrm{SiNi})^{\mathrm{n}+1}
$$$$
\mathrm{K}_{1}{ }^{3} \mathrm{~N}_{1}{ }^{3}+\mathrm{P}_{1}{ }^{3} \mathrm{~N}_{1}{ }^{3}=\mathrm{S}_{1}{ }^{4} \mathrm{~N}_{1}{ }^{4}
$$$$
12^{3} \mathrm{~N}_{1}^{3}+4^{3} \mathrm{~N}_{1}^{3}=4^{4} \mathrm{~N}_{1}^{4}
$$$$
\mathrm{N}_{1}=\frac{\mathrm{K} 1^{\mathrm{n}}+\mathrm{P} 1^{\mathrm{n}}}{\mathrm{S} 1^{\mathrm{n}+1}}
$$$$
\mathrm{N}_{1}=\frac{12^{3}+4^{3}}{4^{4}}=7
$$$$
12^{3} 7^{3}+4^{3} 7^{3}=4^{4} 7^{4}
$$$$
84^{3}+28^{3}=28^{4}
$$

\section{B. ANALYSIS FOR THE CASE}

$$
\begin{aligned}
& \mathbf{0} \leq \mathbf{u}, \mathbf{v}<\mathbf{n} \\
& \mathbf{K}^{\mathbf{n}} \mathbf{N}^{\mathbf{n}}+{ }^{\mathbf{P n}+\mathbf{u}} \mathbf{N}^{\mathrm{n}+\mathbf{u}}=\mathbf{S}^{\mathbf{n}}
\end{aligned}
$$

Case 1.

$$
\begin{aligned}
& u \neq v 0 \leq \mathrm{ur}, \\
& \mathrm{u}, \mathrm{v}=\{0, \\
& \text { a) } \mathrm{u}=0
\end{aligned}
$$

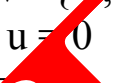

$$
\text { b) } u=1 \text { ) }
$$

Case 2

$$
\mathrm{v} 0 \leq
$$

or $v$ or $b, t h \geq 2$ )

Case 3.

$$
0=\begin{aligned}
& y=m \\
& 0 \leq n-1
\end{aligned}
$$

ANALYSIS CASE 1

a)

$$
\begin{aligned}
& \mathrm{u}=0 \\
& \mathrm{v}=1
\end{aligned}
$$


$\mathrm{K}^{\mathrm{n}} \mathrm{N}^{\mathrm{n}}+\mathrm{P}^{\mathrm{n}+\mathrm{u}} \mathrm{N}^{\mathrm{n}+\mathrm{u}}=\mathrm{N}^{\mathrm{n}+\mathrm{v}}$

$K^{n} \mathbf{N}^{n}+P^{n} N^{n}=\mathbf{N}^{n+1}$

$\mathrm{N}^{\mathrm{n}}\left(\mathrm{K}^{\mathrm{n}}+\mathrm{P}^{\mathrm{n}}\right)=\mathrm{N}^{\mathrm{n}} \mathrm{N}$

$\mathbf{N}=\mathbf{K}^{\mathbf{n}}+\mathbf{P}^{\mathbf{n}} \quad ; \quad \mathrm{n} \geq 3$

$\mathrm{N}$ will be positive integer if $\mathrm{K}$ and $\mathrm{P}$ positive integer $\geq 1$

b)

$$
\begin{aligned}
& \mathrm{u}=1 \\
& \mathrm{v}=0
\end{aligned}
$$

$\mathrm{K}^{\mathrm{n}} \mathrm{N}^{\mathrm{n}}+\mathrm{P}^{\mathrm{n}+\mathrm{u}} \mathrm{N}^{\mathrm{n}+\mathrm{u}}=\mathrm{N}^{\mathrm{n}+\mathrm{v}}$

$\mathrm{K}^{\mathrm{n}} \mathbf{N}^{\mathrm{n}}+\mathrm{N}^{\mathrm{n}+1}=\mathrm{S}^{\mathrm{n}} \mathbf{N}^{\mathrm{n}}$

$\mathrm{N}^{\mathrm{n}}\left(\mathrm{K}^{\mathrm{n}}+\mathrm{N}\right)=\mathrm{S}^{\mathrm{n}} \mathrm{N}^{\mathrm{n}}$

$\mathbf{N}=\mathbf{S}^{\mathbf{n}}-\mathbf{K}^{\mathbf{n}} \quad ; \quad \mathrm{n} \geq 3$

$\mathrm{N}$ will be positive integer if $\mathrm{S}>\mathrm{K}$ and $\mathrm{S}$ and $\mathrm{K}$ positive integer $\geq 1$

This is the solution and proof of Beal's Conjec ur?.

ANALYSIS CASE 2

$\mathrm{A}^{\mathrm{x}}+\mathrm{B}^{\mathrm{y}}=\mathrm{C}^{\mathrm{z}}$

For $\mathrm{u} \neq \mathrm{v} ; 0 \leq \mathrm{u},<\mathrm{h}(\mathrm{u}$ or $\mathrm{v}, \mathrm{r}$ both $\geq 2)$

$\mathrm{K}^{\mathrm{n}} \mathrm{N}^{\mathrm{n}}+\mathrm{P}^{\mathrm{n}+\mathrm{u}} / \mathrm{N}+\mathrm{u}=\mathrm{S}^{\mathrm{n}+\mathrm{v}} \mathrm{N}^{\mathrm{n}} / /: \mathrm{N}^{\mathrm{n}}$

$\mathrm{K}^{\mathrm{n}}+\mathrm{P}^{\mathrm{n}+\mathrm{u}} \mathrm{N}$

$\mathbf{K}^{\mathrm{n}} \mathbf{P}^{\mathrm{n}-} \mathbf{N}^{\mathrm{u}}-\mathrm{y}^{\mathrm{v}} \mathbf{N}^{\mathrm{v}}=\mathbf{0}$

If $\mathrm{x}, \mathrm{y}$, and $\mathrm{a}$ all different in case $0 \leq \mathrm{u}, \mathrm{v}<\mathrm{n},(\mathrm{u}$ or $\mathrm{v}$ or both $\geq 2 ; \mathrm{u} \neq \mathrm{v}$ ) then it is not possible that common prime factor $\mathrm{N}$ and $\mathrm{A}, \mathrm{B}$, and $\mathrm{C}$ are all integers simultaneously, except for the particular case where:

$\begin{array}{ll}\underline{\mathrm{v}=2} & \mathrm{~K}=2 \\ \underline{\mathrm{u}=0} & \mathrm{P}=1 \\ \mathrm{n}=3 & \mathrm{~S}=1\end{array}$

Then equation (1) takes the form 


$$
\begin{aligned}
& \mathrm{K}^{\mathrm{n}} \mathrm{N}^{\mathrm{n}}+\mathrm{N}^{\mathrm{n}}=\mathrm{N}^{\mathrm{n}+\mathrm{v}} \\
& \mathrm{K}^{3} \mathrm{~N}^{3}+\mathrm{N}^{3}=\mathrm{N}^{3+2} \\
& \mathrm{~N}^{2}=\mathrm{K}^{3}+1 \\
& \mathrm{~N}^{2}=2^{3}+1=9 \\
& \mathrm{~N}=3 \\
& 2^{3} \mathrm{~N}^{\mathrm{n}}+\mathrm{N}^{\mathrm{n}}=\mathrm{N}^{\mathrm{n}+2} \\
& 2^{3} \mathrm{~N}^{3}+\mathrm{N}^{3}=\mathrm{N}^{5} \\
& 2^{3} 3^{3}+3^{3}=3^{5} \\
& 6^{3}+3^{3}=3^{5}
\end{aligned}
$$

If the equation $\mathbf{2}^{\mathbf{3}} \mathbf{N}^{\mathbf{n}}+\mathbf{N}^{\mathbf{n}}=\mathbf{N}^{\mathbf{n}+\mathbf{2}}$ is multiplied by $\mathbf{N}^{\mathrm{tn}},(\mathrm{t}=1,2,3,4, .$.$) , we obtain afferent \mathrm{n}$ of same equation .

$$
\begin{aligned}
& \text { E.g. } 1 \quad \mathrm{~N}=3 \\
& \mathrm{n}=3 \\
& \mathrm{t}=1 \\
& \operatorname{tn}=1 \times 3=3 \\
& 2^{3} \mathrm{~N}^{\mathrm{n}}+\mathrm{N}^{\mathrm{n}}=\mathrm{N}^{\mathrm{n}+2} / \mathrm{N}^{\mathrm{tn}} \\
& 2^{3} 3^{3}+3^{3}=3^{5} / 3^{3} \\
& 2^{3} 9^{3}+3^{6}=3^{5+3} \\
& 18^{3}+3^{6}=3^{8} \text { or if: } \\
& 3^{6}=9^{3} ; 3^{8}=9^{4} \text { then: } \\
& 18^{3}+9^{3}=9^{4} \\
& \text { E.g. } 2 \mathrm{n}=3 \\
& \mathrm{t}=2 \\
& \mathrm{nt}=2 \times 3=6 \\
& \mathrm{~N}=3 \\
& 2^{3} \mathrm{~N}^{\mathrm{n}}+\mathrm{N}^{\mathrm{n}}=\mathrm{N}^{\mathrm{n}+2} / \mathrm{N}^{\mathrm{tn}} \\
& 2^{3} 3^{3}+3^{3}=3^{5} / 3^{6} \\
& 2^{3} 3^{9}+3^{9}=3^{11} \text { or } 3^{9}=2 \\
& 54^{3}+27^{3}=3^{11} \\
& \text { ANALYSIC-CASE } \\
& \text { 1. } \mathrm{v}=\mathrm{m} \\
& \text { a.1 } \mathrm{K}^{\mathrm{n}+\mathrm{n}}, \mathrm{P}^{\mathrm{n}}+\mathrm{P}^{\mathrm{n}+\mathrm{m}} \mathrm{N}^{\mathrm{n}+\mathrm{m}}=\mathrm{S}^{\mathrm{n}} \mathrm{N}^{\mathrm{n}} \\
& \mathrm{N}^{\mathrm{n}}\left(\mathrm{K}^{\mathrm{n}+\mathrm{m}} \mathrm{N}^{\mathrm{m}}+\mathrm{P}^{\mathrm{n}+\mathrm{m}} \mathrm{N}^{\mathrm{m}}\right)=\mathrm{S}^{\mathrm{n}} \mathrm{N}^{\mathrm{n}} \\
& \mathrm{N}^{\mathrm{m}}\left(\mathrm{K}^{\mathrm{n}+\mathrm{m}}+\mathrm{P}^{\mathrm{n}+\mathrm{m}}\right)=\mathrm{S}^{\mathrm{n}} \\
& N^{m}=\frac{S^{n}}{K^{n+m}+P^{n+m}}
\end{aligned}
$$

For the common factor $\mathrm{N}$ to be a positive integer, the following conditions must be satisfied: $\mathrm{S}^{\mathrm{n}}$ must be divisible by $\mathrm{K}^{\mathrm{n}+\mathrm{m}}+\mathrm{P}^{\mathrm{n}+\mathrm{m}}$ without a remainder, in that case we can write: 
$\mathrm{K}^{\mathrm{n}+\mathrm{m}}+\mathrm{P}^{\mathrm{n}+\mathrm{m}}=\mathrm{S}$

$\mathrm{N}^{\mathrm{m}}=\frac{\mathrm{S}^{\mathrm{n}}}{\mathrm{S}}=\mathrm{S}^{\mathrm{n}-1}$

$\mathrm{N}=\mathrm{S}^{\frac{\mathrm{n}-1}{\mathrm{~m}}}=\mathrm{S}^{\mathrm{q}}$

The following condition must be fulfilled:

$\mathrm{q}=\frac{n-1}{m}$ - must be positive integers $\geq 1$

$\mathrm{K}^{\mathrm{n}+\mathrm{m}} \mathrm{N}^{\mathrm{n}+\mathrm{m}}+\mathrm{P}^{\mathrm{n}+\mathrm{m}} \mathrm{N}^{\mathrm{n}+\mathrm{m}}=\mathrm{S}^{\mathrm{n}} \mathrm{N}^{\mathrm{n}}$

$\mathrm{q}=\frac{n-1}{m}=\frac{3-1}{2}=1$

$\mathrm{S}=\mathrm{K}^{\mathrm{n}+\mathrm{m}}+\mathrm{P}^{\mathrm{n}+\mathrm{m}}=3^{3+2}+2^{3+2}$

$\mathrm{S}=275$

$\mathrm{N}=\mathrm{S}^{\frac{\mathrm{n}-1}{\mathrm{~m}}}=275^{\frac{3-1}{2}}=275$

$\mathrm{K}^{3+2} \mathrm{~N}^{3+2}+\mathrm{P}^{3+2} \mathrm{~N}^{3+2}=\mathrm{S}^{3} \mathrm{~N}^{3}$

$3^{3+2} 275^{3+2}+2^{3+2} 275^{3+2}=275^{3} 275^{3}$

$3^{5} 275^{5}+2^{5} 275^{5}=275^{6}$

As we can see equation:

$3^{5} 275^{5}+2^{5} 275^{5}=275^{6}$

becomes equation (1)

$\mathbf{K}^{\mathrm{n}} \mathbf{N}^{\mathbf{n}}+\mathbf{P}^{\mathbf{n}} \mathbf{N}^{\mathbf{n}}=\mathbf{N}^{\mathbf{n}+1}$

For $K=3 \mathrm{n}=5$

$\mathrm{P}=2 \mathrm{v}=1$

$\mathrm{S}=1 \mathrm{u}=0$

$\mathrm{N}=\mathbf{K}^{\mathbf{n}}+\mathbf{P}^{\mathbf{n}}=$

$\mathrm{N}=3^{5}+2^{5}=27$

$3^{5} 275^{5}+2^{5} 27$ ? $275^{6}$

b. $\left.1 \quad \mathrm{~K}^{\mathrm{n}} \mathrm{y}+\mathrm{P}\right)^{\mathrm{n}-\mathrm{m}} \mathrm{N}^{\mathrm{n}+\mathrm{m}} \Rightarrow \mathrm{s}^{\mathrm{n}+\mathrm{m}} \mathrm{N}^{\mathrm{n}+\mathrm{m}}$

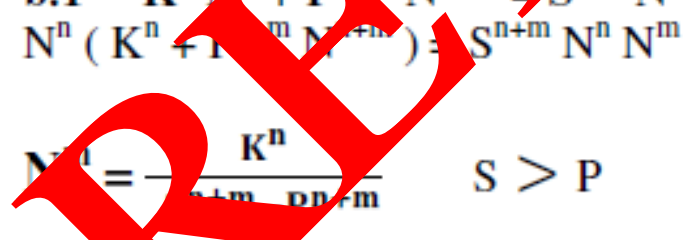

For the con factor $\mathrm{N}$ to be a positive integer, the following conditions must be satisfied:

$\mathrm{K}^{\mathrm{n}}$ must be dirisible by $\mathrm{S}^{\mathrm{n}+\mathrm{m}}-\mathrm{P}^{\mathrm{n}+\mathrm{m}}$ without a remainder, in that case we can write:

$$
\begin{aligned}
& \mathrm{S}^{\mathrm{n}+\mathrm{m}}-\mathrm{P}^{\mathrm{n}+\mathrm{m}}=\mathrm{K} \quad \mathrm{S}>\mathrm{P} \\
& \mathrm{N}=\mathrm{K}^{\frac{\mathrm{n}-1}{\mathrm{~m}}}=\mathrm{K}^{\mathrm{q}}
\end{aligned}
$$

The following condition must be fulfilled:

$\mathrm{q}=\frac{n-1}{m}$ - must be positive integer $\geq 1$

E.g $1 \mathrm{n}=3$

$\mathrm{m}=1$ 


$$
\begin{aligned}
& \mathrm{S}=3 \\
& \quad \mathrm{P}=2 \\
& \mathrm{q}=\frac{n-1}{m}=\frac{3-1}{1}=2 \\
& \mathrm{~K}^{\mathrm{n}} \mathrm{N}^{\mathrm{n}}+\mathrm{P}^{\mathrm{n}+\mathrm{m}} \mathrm{N}^{\mathrm{n}+\mathrm{m}}=\mathrm{S}^{\mathrm{n}+\mathrm{m}} \mathrm{N}^{\mathrm{n}+\mathrm{m}} \\
& \mathrm{N}^{\mathrm{m}}=\mathrm{K}^{\mathrm{n}-1} \\
& \mathrm{~N}=\mathrm{K}^{2} \\
& \mathrm{~K}=\mathrm{S}^{\mathrm{n}+\mathrm{m}}-\mathrm{P}^{\mathrm{n}+\mathrm{m}} \\
& \mathrm{K}=3^{4}-2^{4}=65 \\
& \mathrm{~N}=\mathrm{K}^{2}=65^{2} \\
& 65^{3}\left(65^{2}\right)^{3}+2^{3+1}\left(65^{2}\right)^{3+1}=3^{3+1}\left(65^{2}\right)^{3+1} \\
& 65^{9}+2^{4} 65^{8}=3^{4} 65^{8} \\
& 65^{4} 65^{5}+2^{4} 65^{4} 65^{4}=3^{4} 65^{4} 65^{4} \quad /: 65^{4} \\
& 65^{5}+2^{4} 65^{4}=3^{4} 65^{4} \\
& \mathbf{6 5}^{5}+130^{4}=195^{4}
\end{aligned}
$$

As we can see equation $2^{4} 65^{4+}+65^{5}=3^{4} 65^{4}$ $130^{4}+65^{5}=195^{4}$ becomes equation (2)

$$
\mathbf{K}^{\mathrm{n}} \mathbf{N}^{\mathrm{n}}+\mathrm{N}^{\mathrm{n}+1}=\mathrm{S}^{\mathrm{n}} \mathbf{N}^{\mathrm{n}}
$$

For: $K=2 \mathrm{n}=4$

$$
\begin{aligned}
& \mathrm{S}=3 \mathrm{u}=1 \\
& \mathrm{P}=1 \mathrm{v}=0
\end{aligned}
$$$$
\mathbf{N}=\mathbf{S}^{\mathrm{n}}-\mathbf{K}^{\mathrm{n}}
$$$$
\mathrm{N}=3^{4}-2^{4}=65
$$$$
2^{4} 65^{4}+65^{5}=3^{4} 65^{4}
$$

E.g.2. $\mathrm{n}=3$ $\mathrm{m}=2$ $\mathrm{S}=3$$$
\mathrm{P}=2
$$

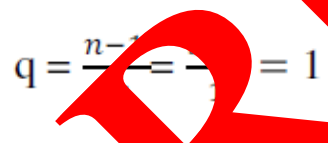

$\mathrm{K}^{\mathrm{n}} \mathrm{N}^{\mathrm{n}}+{ }^{+\mathrm{m}} \mathrm{N}^{\mathrm{n}+\mathrm{m}}=\mathrm{S}^{\mathrm{n}+\mathrm{m}} \mathrm{N}^{\mathrm{n}+\mathrm{m}}$

$\mathrm{N}^{\mathrm{m}}=\mathrm{K}^{\mathrm{n}-1}$

$\mathrm{N}=\mathrm{K}$

$\mathrm{K}=\mathrm{S}^{\mathrm{n}+\mathrm{m}}-\mathrm{P}^{\mathrm{n}+\mathrm{m}}$

$\mathrm{K}=3^{5}-2^{5}=211$

$\mathrm{N}=\mathrm{K}=211$

$211^{3} 211^{3}+2^{3+2} 211^{3+2}=3^{3+2} 211^{3+2}$

$2^{5} 211^{5}+211^{6}=3^{5} 211^{5}$

$422^{5}+211^{6}=633^{5}$ 
As we can see equation:

$$
2^{5} 211^{5}+211^{6}=3^{5} 211^{5}
$$

becomes equation (2)

$$
\begin{aligned}
& \mathbf{K}^{\mathrm{n}} \mathbf{N}^{\mathrm{n}}+\mathrm{N}^{\mathrm{n}+1}=\mathrm{S}^{\mathrm{n}} \mathbf{N}^{\mathrm{n}} \\
& \text { For } \mathrm{K}=2 \mathrm{n}=5 \\
& \mathrm{P}=1 \quad \mathrm{u}=1 \\
& \mathrm{~S}=3 \quad \mathrm{v}=0 \\
& \mathrm{~N}=\mathrm{S}^{5}-\mathrm{K}^{5} \\
& \mathrm{~N}=3^{5}-2^{5} \\
& \mathrm{~N}=211 \\
& 2^{5} 211^{5}+211^{6}=3^{5} 211^{5} \\
& \text { 2) } \mathrm{u}=\mathrm{v}=\mathrm{m} ; \mathrm{m}=0 \\
& \mathrm{~K}^{\mathrm{n}} \mathrm{N}^{\mathrm{n}}+\mathrm{P}^{\mathrm{n}+\mathrm{m}} \mathrm{N}^{\mathrm{n}+\mathrm{m}}=\mathrm{S}^{\mathrm{n}+\mathrm{m}} \mathrm{N}^{\mathrm{n}+\mathrm{m}} \\
& \mathrm{K}^{\mathrm{n}} \mathrm{N}^{\mathrm{n}}+\mathrm{P}^{\mathrm{n}} \mathrm{N}^{\mathrm{n}}=\mathrm{S}^{\mathrm{n}} \mathrm{N}^{\mathrm{n}} \\
& \mathrm{K}^{\mathrm{n}}+\mathrm{P}^{\mathrm{n}}=\mathrm{S}^{\mathrm{n}} \text { or } \\
& A^{n}+B^{n}=C^{n} \text { for } n>2
\end{aligned}
$$

We get the equation for FERMAT'S CONJECTURF

\section{Or Fermat's Last Theorem}

Following the Theorem 1: $x, y$, and $z$ can never be a qual or $d$ fferent simultaneously, for $x$, $y$, and $z>2$

The equation $A^{n}+B^{n}=C^{n}$ for $n>2$ has which at least one of the variables is zero.

For $n=2$ we get Pythagorean theorem.

\section{EXAMPLES AND DISCUS TN}

EXAMPLE: 1

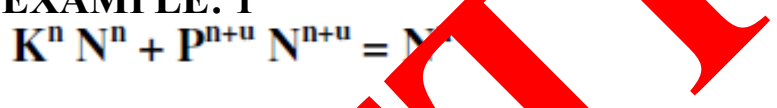

$\mathrm{n} \geq 3 ; \mathrm{K}, \mathrm{P}, \mathrm{S} \geq 1, \mathrm{U} \neq \mathrm{v} ; \mathrm{u}, \mathrm{v}=$

a)

$$
\mathrm{v}=1
$$

$\mathrm{K}^{\mathrm{n}} \mathrm{N}^{\mathrm{n}} \quad \mathrm{P}^{\mathrm{n}} \mathrm{N}=\mathrm{N}^{\mathrm{n}+1}$

$\mathbf{N}=\mathbf{K}$

$$
\text { E.g.1. } \begin{array}{ll}
\mathrm{K}=3 & \underline{\mathrm{n}=3} \\
\mathrm{P}=2 & \underline{\mathrm{u}=0} \\
\mathrm{~S}=1 & \mathrm{v}=1
\end{array}
$$

$$
\begin{aligned}
& \mathbf{K}^{n} N^{n}+P^{n} N^{n}=N^{n+1} \\
& N=K^{n}+P^{n}
\end{aligned}
$$

$\mathrm{N}=3^{3}+2^{3}$

$\mathrm{N}=35$

$3^{3} 35^{3}+2^{3} 35^{3}=35^{4}$

$105^{3}+70^{3}=35^{4}$ 
If $\mathrm{N}$ is common factor:

$\mathrm{N}=\mathrm{N}_{1} \times \mathrm{N}_{2} \times \ldots$

$\mathrm{N}_{1}=\frac{\mathrm{K} 1^{\mathrm{n}}+\mathrm{P} \mathbf{1}^{\mathrm{n}}}{\mathrm{S} \mathbf{1}^{\mathrm{n}+\mathbf{1}}}$

$\mathrm{N}_{1}=\mathrm{f}\left(\mathrm{K}_{1}, \mathrm{P}_{1}, \mathrm{~S}_{1}\right)-$ common prime factor

$\mathrm{K}_{1}, \mathrm{P}_{1}, \mathrm{~S}_{1} \geq 1$

$\mathrm{N}=35$ is common factor

$\mathrm{N}=5 \times 7=\mathrm{N}_{1} \times \mathrm{N}_{2}$

$\mathrm{N}_{1}=5$ and $\mathrm{N}_{2}=7$ are common prime factors

And we get the same if we write:

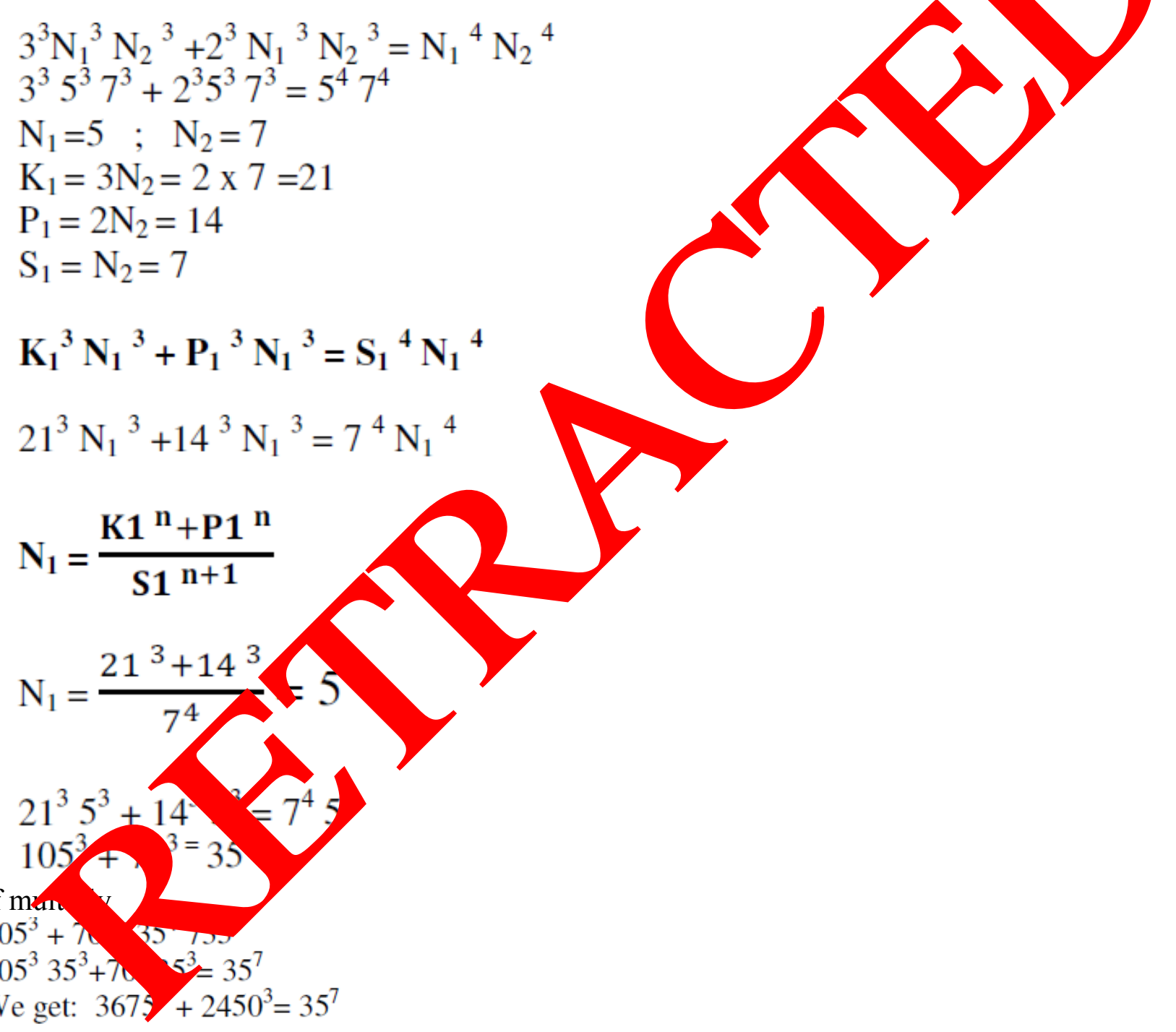

We get: $367 \%+2450^{3}=35^{7}$

Where is:

$\mathrm{N} 1=5$ common prime factor 
$\begin{array}{lll}\text { E.g.2. } & \mathrm{K}=2 & \underline{\mathrm{n}=3} \\ & \mathrm{P}=2 & \mathrm{u}=0 \\ & \mathrm{~S}=1 & \mathrm{v}=1\end{array}$

$\mathbf{K}^{\mathrm{n}} \mathbf{N}^{\mathrm{n}}+\mathrm{P}^{\mathrm{n}} \mathbf{N}^{\mathrm{n}}=\mathrm{N}^{\mathrm{n}+1}$

$\mathbf{N}=\mathbf{K}^{\mathrm{n}}+\mathbf{P}^{\mathrm{n}}$

$\mathrm{N}=2^{3}+2^{3}$

$\mathrm{N}=16$

$2^{3} 16^{3}+2^{3} 16^{3}=16^{4}$

$32^{3}+32^{3}=16^{4}$ or

$2^{15}+2^{15}=4^{8}$

$\mathrm{N}_{\mathrm{i}}=2$ is common prime factor

For: $\mathrm{n} \geq 3$

$\mathrm{u}=0 ; \mathrm{v}=1$

$\mathrm{K}, \mathrm{P}, \mathrm{S} \geq 1$

Or: $\mathrm{Ni}=\mathrm{f}\left(\mathrm{K}_{\mathrm{i}}, \mathrm{P}_{\mathrm{i}}, \mathrm{S}_{\mathrm{i}}\right)$-common prime factor $\mathrm{K}_{\mathrm{i}}, \mathrm{P}_{\mathrm{i}}, \mathrm{S}_{\mathrm{i}}, \geq 1$ - positive integ

Multiple equation with $\mathrm{N}^{\text {nt }}$ or $\mathrm{N}^{\text {int }}$

$\mathrm{t}=\{1,2,3, \ldots\}$,

we obtain infinite number of solutions, in different form.

EXAMPLE: 2

$\mathbf{K}^{\mathrm{n}} \mathbf{N}^{\mathrm{n}}+\mathbf{P}^{\mathrm{n}+\mathrm{u}} \mathbf{N}^{\mathrm{n}+\mathrm{u}}=\mathbf{N}^{\mathrm{n}+\mathrm{v}}$

$\mathrm{n} \geq \mathbf{3} ; \mathrm{K}, \mathrm{P}, \mathrm{S} \geq \mathbf{1} ; \mathrm{u} \neq \mathrm{v} ; \mathrm{u}, \mathrm{v}=\{0,1\}$

$$
\text { b) } \begin{aligned}
& \mathbf{u}=1 \\
& \mathbf{v}=\mathbf{0}
\end{aligned}
$$

$\mathbf{K}^{\mathrm{n}} \mathbf{N}^{\mathrm{n}}+\mathbf{N}^{\mathrm{n}+1}=\mathbf{S}^{\mathrm{n}} \mathbf{N}^{\mathrm{n}}$
$\mathbf{N}=\mathbf{S}^{\mathrm{n}}-\mathbf{K}^{\mathrm{n}} \quad \mathrm{S}>\mathrm{K}$

E.g.1. $\mathrm{K}=1$

$\mathrm{S}=2$

$\mathrm{P}=1$

$\mathrm{K}^{\mathrm{n}} \mathbf{N}^{\mathrm{n}}+\mathbf{N}^{\mathrm{n}+1}=\mathrm{S}$

$\mathrm{N}=\mathrm{S}^{\mathrm{n}}-\mathbf{n}$

$\mathrm{N}=2$

$\mathrm{N}=15$

$15^{4}+15^{5}=2.5^{4}$

$15^{4}+15^{5}=30^{4}$

For $\mathrm{N}$ is common factor:

$\mathrm{N}=\mathrm{N}_{1} \mathrm{~N}_{2} \ldots$

$\mathrm{N}_{1}=\frac{S 1^{\mathrm{n}}-K 1^{\mathrm{n}}}{\mathrm{P}^{\mathrm{n}+1}}$

$\mathrm{N}=15$ is common factor

$\mathrm{N}=5 \times 3=\mathrm{N}_{1} \times \mathrm{N}_{2}$

$\mathrm{N}_{1}=5$ and $\mathrm{N}_{2}=3$ are common prime factors. And we get the same if we write: 


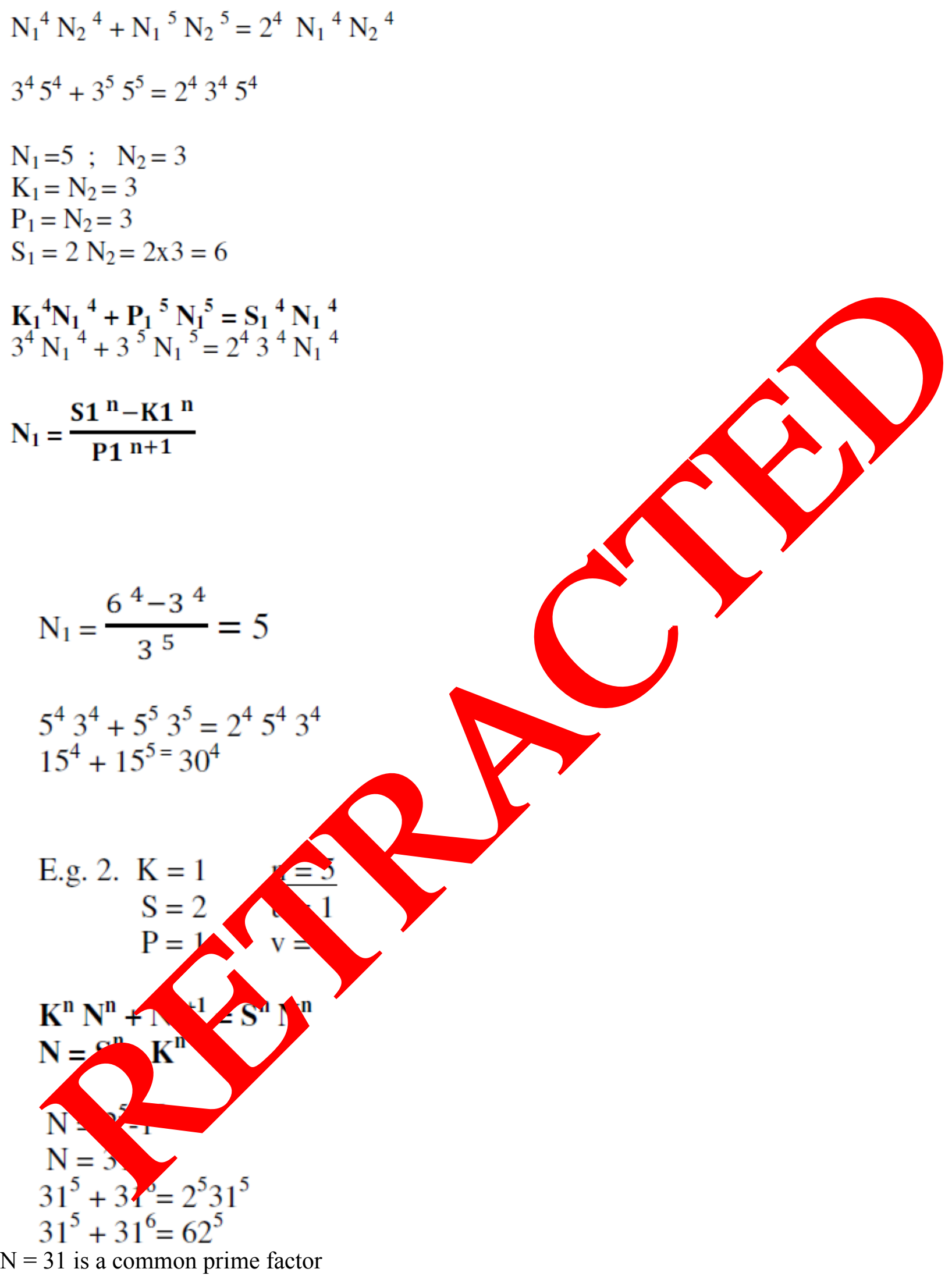


$\begin{array}{lll}\text { E.g.3. } & \mathrm{K}=3 & \underline{\mathrm{n}=4} \\ \mathrm{~S}=5 & \mathrm{u}=1 \\ \mathrm{P}=1 & \mathrm{v}=0\end{array}$

$\mathrm{K}^{\mathrm{n}} \mathrm{N}^{\mathrm{n}}+\mathrm{N}^{\mathrm{n}+1}=\mathrm{S}^{\mathrm{n}} \mathrm{N}^{\mathrm{n}}$

$\mathrm{N}=\mathrm{S}^{\mathbf{n}}-\mathrm{K}^{\mathbf{n}}$

$\mathrm{N}=5^{4}-3^{4}$

$\mathrm{N}=544$

$3^{4} 544^{4}+544^{5}=5^{4} 544^{4}$

If: $\quad 544=32 \times 17$

$3^{4} 32^{4} 17^{4}+32^{5} 17^{5}=5^{4} 32^{4} 17^{4}$

$96^{4} 17^{4}+32^{5} 17^{5}=160^{4} 17^{4}$

$\mathrm{K}_{1}{ }^{\mathrm{n}} \mathrm{N}_{1}{ }^{\mathrm{n}}+\mathrm{P}_{1}{ }^{\mathrm{n}+1} \mathrm{~N}_{1}{ }^{\mathrm{n}+1}=\mathrm{S}_{1}{ }^{\mathrm{n}} \mathrm{N}_{1}{ }^{\mathrm{n}}$

$\mathbf{N}_{\mathbf{1}}=\frac{\mathbf{S} \mathbf{1}^{\mathbf{n}}-\mathbf{K} \mathbf{1}^{\mathbf{n}}}{\mathbf{P} \mathbf{1}^{\mathbf{n + 1}}}=\frac{160^{4}-96^{4}}{32^{5}}$

$\mathrm{N}_{1}=17$

$3^{4} 32^{4} 17^{4}+32^{5} 17^{5}=5^{4} 32^{4} 17^{4} \quad$ I: $32^{4}$

$3^{4} 17^{4}+32 \times 17^{5}=5^{4} 17^{4}$

$32=2^{5}$

$3^{4} 17^{4}+2^{5} 17^{5}=5^{4} 17^{4}$

$\mathrm{K}_{2}{ }^{\mathrm{n}} \mathrm{N}_{2}{ }^{\mathrm{n}}+\mathrm{P}_{2}{ }^{\mathrm{n}+1} \mathrm{~N}_{2}{ }^{\mathrm{n}+1}=\mathrm{S}_{2}{ }^{\mathrm{n}} \mathrm{N}_{2}$

$\mathrm{K}_{2}=3 ; \mathrm{P}_{2}=2 ; \mathrm{S}_{2}=5 ; \mathrm{n}=4$

$\mathbf{N}_{2}=\frac{S 2^{\mathbf{n}}-K 2^{\mathbf{n}}}{\mathbf{P} 2^{\mathbf{n}+1}}=\frac{5^{4}-3^{4}}{2^{5}}=17$

$\mathrm{N}^{2}=17$ is a common prime actor

For: $\mathrm{n} \geq 3 ; \mathrm{u}=1$

Or $\mathrm{Ni}=\mathrm{f}(\mathrm{Ki}, \mathrm{Pi}, \mathrm{l})$ is a common $\mathrm{p}$ me factor

$\mathrm{Ki}, \mathrm{Pi}, \mathrm{Si}$ are po ve $\mathrm{j}$,egers 1

Multiple on de equ on with Nnt or Nint $t=\{1,2,3 \ldots\}$, we get a different form of equation and obtain infiy te number of solutions.

From the an. sis above we can conclude that every solution is a part off and belongs to the solution set of equations (1), (1.1), (2) and (2.1), which are solution of the Beal Conjecture. 
D. MORE EXAMPLES

of applications of equations (1), (1.1), (2) and (2.1) as solutions of Beal Conjecture

1. $\mathrm{K}=1 \underline{\mathrm{n}=5}$

$\mathrm{P}=1 \quad \mathrm{u}=0$

$\mathrm{S}=1 \quad \mathrm{v}=1$

$\mathrm{K}^{\mathrm{n}} \mathrm{N}^{\mathrm{n}}+\mathrm{P}^{\mathrm{n}} \mathrm{N}^{\mathrm{n}}=\mathrm{N}^{\mathrm{n}+1}$

$\mathbf{N}=\mathbf{K}^{\mathrm{n}}+\mathbf{P}^{\mathrm{n}}$

$\mathrm{N}=1+1=2$

$2^{5}+2^{5}=2^{6}$ or

$2^{5}+2^{5}=4^{3}$

$\underline{\mathrm{N}=2}$

2. $\mathrm{K}=3 \quad \underline{\mathrm{n}=3}$

$\mathrm{P}=3 \quad \mathrm{u}=0$

$\mathrm{S}=1 \quad \mathrm{v}=1$

$\mathrm{K}^{\mathrm{n}} \mathrm{N}^{\mathrm{n}}+\mathrm{P}^{\mathrm{n}} \mathrm{N}^{\mathrm{n}}=\mathrm{N}^{\mathrm{n}+1}$

$\mathbf{N}=\mathbf{K}^{\mathbf{n}}+\mathbf{P}^{\mathbf{n}}$

$\mathrm{N}=3^{3}+3^{3}$

$\mathrm{N}=54$

$3^{3} 54^{3}+3^{3} 54^{3}=54^{4}$

$3^{3} 2^{3} 27^{3}+3^{3} 2^{3} 27^{3}=2^{4} 27^{4}$

$162^{3}+162^{3}=54^{4}$

3. $\mathrm{K}=4 \underline{\mathrm{n}=3}$

$\mathrm{P}=1 \quad \mathrm{u}=0$

$\mathrm{S}=1 \quad \mathrm{v}=1$

$\mathrm{K}^{\mathrm{n}} \mathrm{N}^{\mathrm{n}}+\mathrm{P}^{\mathrm{n}} \mathrm{N}^{\mathrm{n}}=\mathrm{P}^{+1}$

$\mathrm{N}=\mathbf{K}^{\mathrm{n}}+\mathrm{P}^{\mathbf{n}}$

$\frac{\mathrm{N}_{1}=\mathbf{3}}{\mathrm{N}_{2}=2}$

$\mathrm{N}=\mathrm{K}^{\mathrm{n}}+\mathrm{P}$

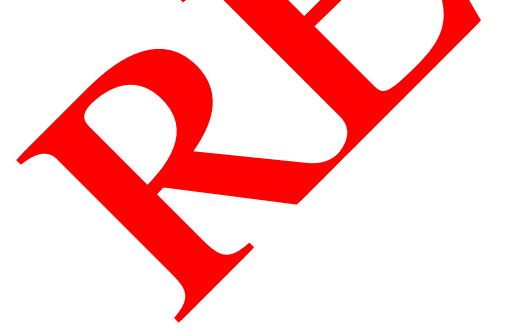


$\mathrm{N}=4^{3}+1^{3}$

$\mathrm{N}=65$

$4^{3} 65^{3}+65^{3}=65^{4}$

$4^{3} 13^{3} 5^{3}+13^{3} 5^{3}=13^{4} 5^{4}$

$52^{3} 5^{3}+13^{3} 5^{3}=13^{4} 5^{4}$

$260^{3}+65^{3}=65^{4}$ $\frac{\mathbf{N}_{1}=\mathbf{5}}{\mathrm{N}_{2}=13}$

4. $\mathrm{K}=1 \quad \underline{\mathrm{n}=3}$

$\mathrm{P}=1 \quad \overline{\mathrm{u}=1}$

$\mathrm{S}=2 \quad \mathrm{v}=0$

$\mathbf{K}^{\mathrm{n}} \mathbf{N}^{\mathrm{n}}+\mathbf{N}^{\mathrm{n}+1}=\mathrm{S}^{\mathrm{n}} \mathbf{N}^{\mathrm{n}}$

$\mathrm{N}=\mathrm{S}^{\mathrm{n}} \cdot \mathrm{K}^{\mathrm{n}}$

$\mathrm{N}=2^{3}-1$

$\mathrm{N}=7$

$7^{3}+7^{4}=2^{3} 7^{3}$

$7^{3}+7^{4}=14^{3}$

$\mathrm{N}=7$

5. $\mathrm{K}=2 \quad \underline{\mathrm{n}=5}$

$\mathrm{P}=1 \quad \mathrm{u}=0$

$\mathrm{S}=1 \quad \mathrm{~V}=1$

$\mathbf{K}^{\mathrm{n}} \mathbf{N}^{\mathrm{n}}+\mathbf{P}^{\mathrm{n}} \mathbf{N}^{\mathrm{n}}=\mathbf{N}^{\mathrm{n}+1}$

$\mathbf{N}=\mathbf{K}^{\mathrm{n}}+\mathbf{P}^{\mathrm{n}}$

$\mathrm{N}=2^{5}+1$

$\mathrm{N}=33$

$2^{5} 33^{5}+33^{5}=33^{6}$

$2^{5} 3^{5} 11^{5}+3^{5} 11^{5}=$

$6^{5} 11^{5}+3^{5} 11^{5}=$

$66^{5}+33^{5}=33^{6}$

6. $\mathrm{K}=2$

$\mathrm{P}=1$

$\mathrm{S}=1$

$\mathrm{K}<\mathrm{n}$

$\mathrm{N}=5+1$

$\mathrm{N}=63$

$2^{6} 65^{6}+65^{6}=65^{7}$

$2^{6} 5^{6} 13^{6}+5^{6} 13^{6}=5^{7} 13^{7}$

$10^{6} 13^{6}+5^{6} 13^{6}=5^{7} 13^{7}$

$130^{6}+65^{6}=65^{7}$

$\frac{N_{1}=13}{N_{2}=5}$

7. $\mathrm{K}=2 \quad \underline{\mathrm{n}=4}$

$\mathrm{P}=1 \quad \mathrm{u}=0$

$\mathrm{S}=1 \quad \mathrm{v}=1$ 


$$
\begin{aligned}
& \mathbf{K}^{\mathbf{n}} \mathbf{N}^{\mathbf{n}}+\mathbf{P}^{\mathrm{n}} \mathbf{N}^{\mathrm{n}}=\mathbf{N}^{\mathrm{n}+1} \\
& \mathbf{N}=\mathbf{K}^{\mathrm{n}}+\mathbf{P}^{\mathbf{n}} \\
& \mathrm{N}=2^{4}+1 \\
& \mathrm{~N}=17 \\
& 2^{4} 17^{4}+17^{4}=17^{5} \\
& 34^{4}+17^{4}=17^{5}
\end{aligned}
$$

8. $\mathrm{K}=2 \quad \underline{\mathrm{n}}=3$

$\mathrm{P}=1 \quad \mathrm{u}=1$

$\mathrm{S}=3 \quad \mathrm{v}=0$

$\mathrm{K}^{\mathrm{n}} \mathrm{N}^{\mathrm{n}}+\mathrm{N}^{\mathrm{n}+1}=\mathrm{S}^{\mathrm{n}} \mathrm{N}^{\mathrm{n}}$

$\mathrm{N}=\mathrm{S}^{\mathrm{n}} \cdot \mathrm{K}^{\mathrm{n}}$

$\mathrm{N}=3^{3}-2^{3}$

$\mathrm{N}=19$

$2^{3} 19^{3}+19^{4}=3^{3} 19^{3}$

$38^{3}+19^{4}=57^{3}$

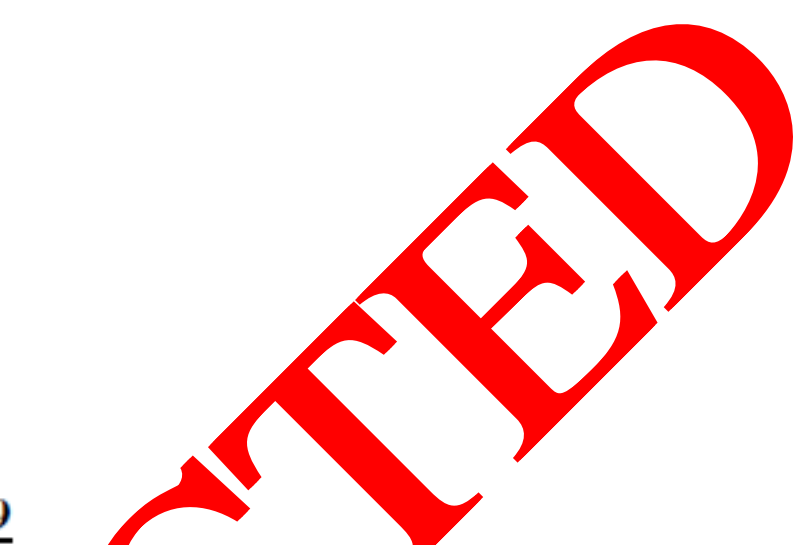

CONCLUSION

Starting from the Beal Conjecture

$A^{x}+B^{y}=C^{z}$ where

$A, B, C, x, y$, and $z$ are common prime factor.

Than equations (1), (1,$(2.1)$, are olutions of the Beal Conjecture for every value of $K$, $P$ and $S \geq 1 ; n \geq 3$; an $(u, v) \quad, 1\}$ including particular case where $(u=0 ; v=2)$, as shown. This is the solutio and proof on Conjecture.

\section{REFERENCES}

[1] Don Bu ys, Prou Beal's Conjecture By: Don Blazys

[2] nar LTorres Di Gregorio, "Proof for the Beal Conjecture and new proof for Fermat's Last The m intre and Applied Mathematics Journal,

[3] pp 14, 55, Published Online September 20, 2013.

[4] Prof. di K. Rama Gandhi and Reuven Tint, Proof of Beal's Conjecture Bulletin of Mathematics Sciences \& Applications pp. 61-64 (2013)

[5] Jamel Ghanouchi, A proof of Beal's Conjecturehal-00710017 version 4 - 22 Jun 2012

[6] Byomkes Chanora Ghosh, The proof the Beal's Conjecture , Calcuta Mathematical Society, EA-374 Sector-1 Salt lake City

[7] Charles William Johnson, A proof and Counterexamples Earth/matrix Editions 4-22 Aug. 2002

[8] Mauldin, R.D,A Generalization of Fermat's Last Theorem: The Beal Conjecture and prize problem, AMS Notices 44, No 11, Dec. 1997, 1436-1437

[9] Raj C Thiagarajan, PhD A Proof to Beal's Conjecture 29. Aug. 2013. www.atoa.com, Rev 5, 14. Jan., page 1 